\title{
Brandl, Werner
}

\section{Lernstand erheben, Lernwirksamkeit feststellen, Lernleistung beurteilen - eine Skizze des Bezugssystems}

Haushalt in Bildung \& Forschung 5 (2016) 4, S. 3-24

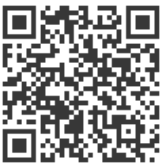

Quellenangabe/ Reference:

Brandl, Werner: Lernstand erheben, Lernwirksamkeit feststellen, Lernleistung beurteilen - eine Skizze des Bezugssystems - In: Haushalt in Bildung \& Forschung 5 (2016) 4, S. 3-24 - URN:

urn:nbn:de:0111-pedocs-203363 - DOI: 10.25656/01:20336

https://nbn-resolving.org/urn:nbn:de:0111-pedocs-203363

https://doi.org/10.25656/01:20336

in Kooperation mit / in cooperation with:

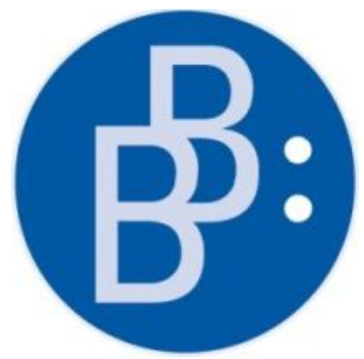

https://www.budrich.de

\section{Nutzungsbedingungen}

Gewährt wird ein nicht exklusives, nicht übertragbares, persönliches und beschränktes Recht auf Nutzung dieses Dokuments. Dieses Dokument is ausschließlich für den persönlichen, nicht-kommerziellen Gebrauch bestimmt. Die Nutzung stellt keine Übertragung des Eigentumsrechts an diesem Dokument dar und gilt vorbehaltlich der folgenden Einschränkungen: Auf sämtlichen Kopien dieses Dokuments müssen alle Urheberrechtshinweise und sonstigen Hinweise auf gesetzlichen Schutz beibehalten werden. Sie dürfen dieses Dokument nicht in irgendeiner Weise abändern, noch dürfen Sie dieses Dokument für öffentliche oder kommerzielle Zwecke vervielfältigen, öffentlich ausstellen, aufführen, vertreiben oder anderweitig nutzen.

Mit der Verwendung dieses Dokuments erkennen Sie die Nutzungsbedingungen an.

\section{Terms of use}

We grant a non-exclusive, non-transferable, individual and limited right to using this document.

This document is solely intended for your personal, non-commercial use. Use of this document does not include any transfer of property rights and it is conditional to the following limitations: All of the copies of this documents must retain all copyright information and other information regarding legal protection. You are not allowed to alter this document in any way, to copy it for public or commercial purposes, to exhibit the document in public, to perform, distribute or otherwise use the document in public.

By using this particular document, you accept the above-stated conditions of use.

\section{Kontakt / Contact:}

\section{peDOCS}

DIPF | Leibniz-Institut für Bildungsforschung und Bildungsinformation Informationszentrum (IZ) Bildung

E-Mail:pedocs@dipf.de

Internet: www.pedocs.de

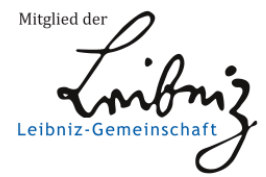




\section{Jahrgang} Heft 4

2016

旁

๓

$\bullet$

号

$\bullet$

돈

용

『্冖

$\frac{\bar{T}}{\Phi}$

$\frac{9}{2}$

정

은

$\stackrel{ \pm}{\frac{1}{2}}$

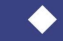

c

$\frac{0}{1}$

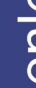

$\xi$

ह

N

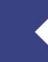

$=$

s

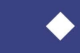

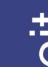

$\frac{2}{2}$

항

ISSN 2193-8806

\section{Haushalt in \\ Forschung}

\section{Lernwirksamkeitsmessung I}

Theoriebildung \& Messinstrumente
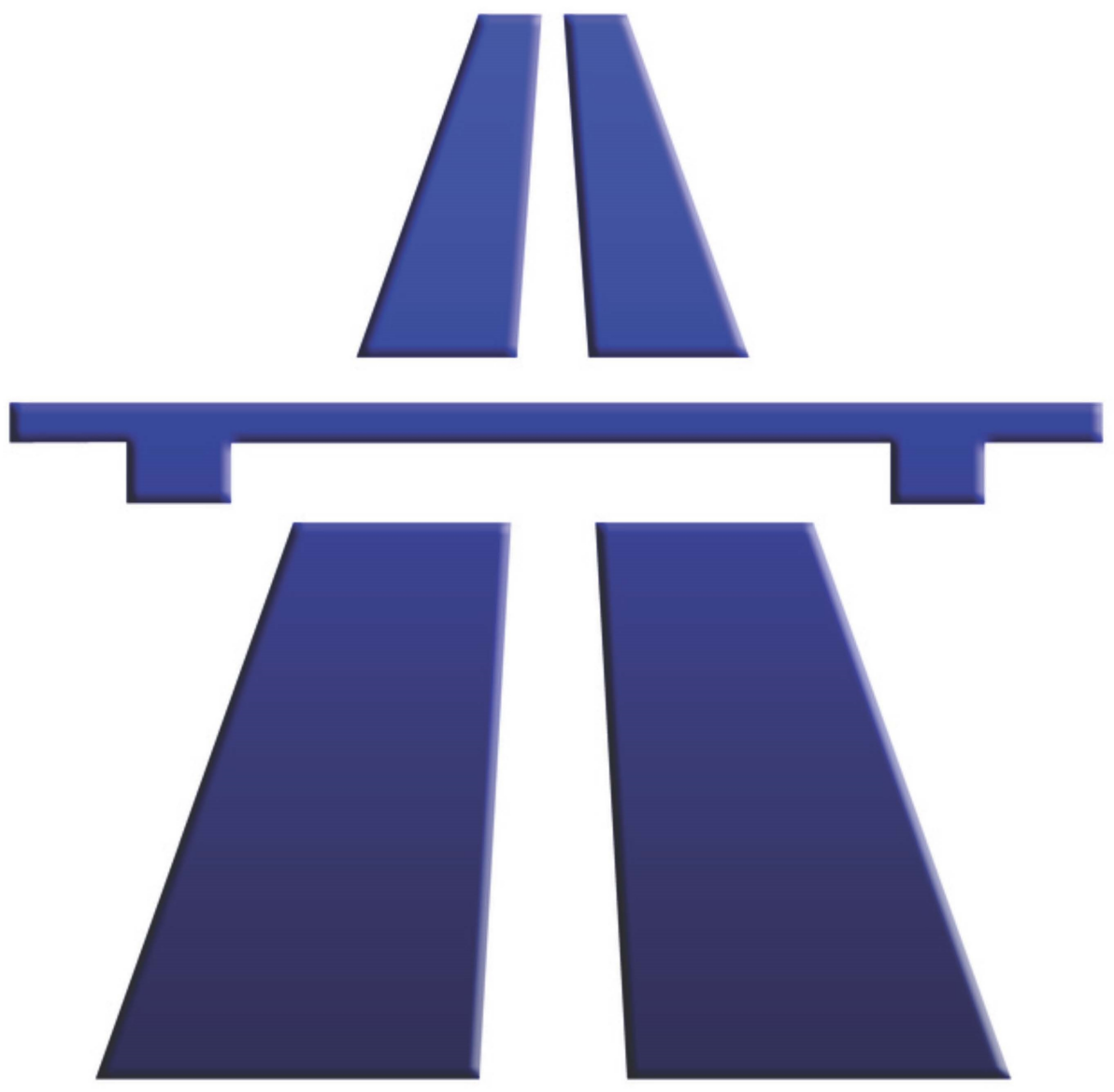


\section{Inhaltsverzeichnis}

Ursula Buchner

Editorial. 2

Werner Brandl

Lernstand erheben, Lernwirksamkeit feststellen,

Lernleistung beurteilen - eine Skizze des Bezugssystems

Gabriele Danninger

Neurodidaktik und Lernwirksamkeit im Lernfeld Ernährung.... 25

Sabine Albert

Die Bedeutung der reflexiven Selbstforschung für die Professionalisierung von Lehrpersonen

Sandro Biaggi \& Claudia Wespi

Professionskompetenzen fördern mit eigenen Videos aus dem Praktikum -

Einblicke in Erfahrungen von Studierenden und Dozierenden

Irmgard Dachtler-Freiler

Neuer Lehrplan - und was nun? Basisüberlegungen zur Lernwirksamkeit

des neuen Lehrplans 61

Martina Überall, Maria Lerchbaumer, Christa Meliss \& Birgit Wild

Nachhaltigkeit schmeckt!

Ursula Buchner \& Maria Magdalena Fritz

Lernen im Fach Gesundheit und Soziales: Was wirkt?

Karin Lindner

Langfristige Auswirkungen von Auslandspraktika

auf die berufliche Entwicklung.

Julia Hirsch

Rezension: Essen und Ernährungsbildung in der KiTa 123 
Werner Brandl

\section{Lernstand erheben, Lernwirksamkeit feststellen, Lernleistung beurteilen - eine Skizze des Bezugssystems}

Lernen und Leistung wird allgemein ein symbiotisches Verhältnis zu gegenseitigem Nutzen zugeschrieben: Ohne mentale Anstrengung kein Lernen und ohne Lernprozesse auch kein Leistungsvermögen. Konzeptionell, strukturell sowie prozessual sind beide jedoch zu unterscheiden und deshalb sind auch die Messung und Beurteilung der Lernleistung von der Erhebung des Lernstandes und der Feststellung der Lernwirksamkeit zu trennen.

Schlüsselwörter: Lernen, Lernstand, Lernwirksamkeit, Lernleistung

\section{Einblick: Theorien - Akteure - Systeme}

Unter dem Stichwort „Gamification“, nämlich dem Einsatz von ,game design elements in non-game contexts“ (Deterding et al., 2011, S. 1), der „Übertragung von spieltypischen Elementen und Vorgängen in spielfremde Zusammenhänge mit dem Ziel der Verhaltensänderung und Motivationssteigerung bei Anwenderinnen und Anwendern" (Gabler Wirtschaftslexikon: Stichwort Gamification) wird neuerdings und wieder einmal - diesmal mit deutlicher Ausrichtung auf smarte Hardware und dementsprechender IT-Unterstützung - ein Anlauf gestartet, „spielend lernen“ als didaktisch-methodischen Dernier Cri zu propagieren. Ein eher unvoreingenommener Blick auf die Vorbereitung, Durchführung und das Ergebnis von (absichtsvollen) Lernprozessen kann dagegen schon etwas ernüchtern!

$\mathrm{Ob}$ es um das selbstgewählte und selbstgesteckte Ziel geht, sich die Sprache für den Griechenland-Urlaub, die Beherrschung eines Instruments zur musikalischen Freizeitgestaltung anzueignen oder darum, dass die in Lehrplänen festgehaltenen und für notwendig befundenen Lerninhalte, z. B. des Pythagoras, der deutschen Grammatik oder der Grundrechenarten von entsprechend ausgebildetem Lehrpersonal den Lernenden angeboten werden: Neben allen (auch von Außenstehenden bemerkbaren) Maßnahmen - von der Auswahl der Materialien, der Lernmittel, die Wahl von Ort und Zeit, das Ausmaß des Aufwands an Zeit etc. - sind es vor allem die (dem Lernenden allein introspektiv zugänglichen) mentalen Anstrengungen, das eigene und/oder von anderen Gesehene, Gehörte, Gefühlte, Gedachte, Geschriebene, Gesagte sich gedanklich kompatibel und damit für sich verfügbar zu machen ${ }^{1}$ (Stolperstei- 


\section{Lernstand - Lernwirksamkeit - Lernleistung}

ne, Hindernisse, Irr- und Umwege, Sackgassen etc. im Wahrnehmen, Denken und Erkennen durchaus inklusive), die deutlich erkennen lassen, dass Lernen ohne Aufwand, Anstrengung, Bemühung, letztlich als Ausdruck pädagogisch-psychologisch gefasster Leistung ${ }^{2}$ nicht zu haben ist, kurz: „Betrachte Lernen als harte Arbeit!“ (Hattie \& Zierer, 2016).

Wie immer man auch Lernen theoretisch fasst und definiert, als gemeinsamer Nenner steht eine quantitative und/oder qualitative Änderung von Wissen und Können, der Fähigkeiten und Fertigkeiten kognitiver, motivationaler, volitionaler, aktionaler, emotionaler, sozialer Provenienz beim Lernenden stets im Fokus der Betrachtung. Und ein ebenso gemeinsames Denkmuster stellen die Fragen nach den dafür notwendigen äußeren Maßnahmen - räumlicher, zeitlicher, formaler, didaktischmethodischer, medialer Art - zur Förderung eines ,Mehr und Besseren ' dar, sowie der Möglichkeiten, dieses auch nachweisbar - beleg- und messbar - zu dokumentieren und zu interpretieren.

Die Stichhaltigkeit einer solchen Dokumentation und Interpretation bedarf eines differenzierten Blicks auf die Facetten der Konzept-, Struktur-, Prozess- und Ergebnisqualitäten aus der

- Theorieperspektive: Theorien über das Lernen, Lehren, Leistung, Methoden, Medien

- Akteursperspektive: Selbst- und Rollenverständnis der Lernenden ${ }^{3}$ und Lehrenden, Lern- und Lehrstrategien

- Systemperspektive: Strukturvorgaben/Rahmenbedingungen, Curricula/ Lehrpläne/Module

\section{Lernen - Bildung}

Was ist Bildung, wenn sie nicht Lernen ist? Und was ist Lernen, wenn es sich nicht um Bildung handelt?

(Nohl, von Rosenberg \& Thomsen, 2015, S. 9)

In der Diskussion um die Kompetenzen im Kontext der Leistungsmessung in Schulen (Weinert, 2014) und entsprechender „Kompetenzmodelle zur Erfassung individueller Lernergebnisse und zur Bilanzierung von Bildungsprozessen“ (vgl. Fleischer et al., 2013) ist vielfach Unbehagen und Missverständliches geäußert worden: Zum einen wurde (und wird) - insbesondere aus der Schulpraxis kommend - vermutet, dass mit dem Begriff Kompetenz lediglich quasi ein „Etikettenschwindel“ durch schlichtes Umetikettieren der einst als Lernziele gefassten Anforderungen stattfinde, es sich also lediglich um dasselbe mit anderer Bezeichnung handle oder - insbesondere aus den korrespondierenden Fachwissenschaften stammend - damit gar ein Verzicht auf Sachlichkeit und Fachlichkeit einhergehe und zum anderen die Befürchtung - aus 


\section{Lernstand - Lernwirksamkeit - Lernleistung |}

der Pädagogik, der Bildungstheorie, der Allgemeinen Didaktik geäußert - verbunden wird, dass das als Bildung hochgehandelte Ziel aller Bestrebungen und Bemühungen darüber verlustig gehe.

In der Tat sollte man den unterschiedlichen Fokus, der mit Lernprozessen, Bildungsprozessen und Kompetenzentwicklung verbunden ist, nicht verwischen: Lernen bezeichnet die ,aneignende Auseinandersetzung mit Ausschnitten aus der Welt sogenannten Lerngegenständen oder -themen, wobei hier sich auch die gegenstandsoder fertigkeitsbezogenen Horizonte der Lernenden transformieren können. Bildung hingegen bezeichnet die Transformation der Selbst- und Weltreferenz in ihrer Gesamtheit“ (Nohl, von Rosenberg \& Thomsen, 2015, S. 207).

- Beim Lernen „verändern sich nur Ausschnitte von Selbst und Welt, denn Lernen ist stets auf ,bestimmte Fertigkeiten' bezogen und damit ,Lernen von etwas' wobei nicht nur Können, sondern auch explizites Wissen angeeignet wird" (Nohl, von Rosenberg \& Thomsen, 2015, S. 256).

- Das Bildungsgeschehen bezieht sich „stets auf die zentralen ,Lebensorientierungen' eines Menschen, d. h. auf seine Selbst- und Weltreferenz in ihrer Totalität“" (Nohl, von Rosenberg \& Thomsen, 2015, S. 256).

In der Diskussion um den „Wechsel“ vom Bildungsgedanken zum Kompetenzbegriff zur Beschreibung solch zentraler Lebensorientierungen wird zwar dann und wann lediglich eine „semantische Verschiebung“ in den „Selbstbeschreibungen des Erziehungssystems" (Brosziewski, 2010) vermutet und auch die Frage gestellt, inwieweit Kompetenzmodelle (Kompetenzskalen, Kompetenzstufenmodelle, Kompetenzstrukturmodelle und Kompetenzdiagnosemodelle) überhaupt geeignet seien, auch kognitive Prozesse abzubilden (vgl. Leuders \& Sodian, 2013). Vielleicht liegt es auch daran, dass die (zumindest vermutete) Differenz von Wissen und Kompetenz doch nicht so einfach zu bestimmen ist, sich als vielmehr komplexes Gefüge erweist: Blömeke, Gustafsson und Shavelson plädieren dementsprechend dafür, von einer "unproductive dichotomy view of competence" - nämlich entweder Kompetenz oder Performanz - Abstand zu nehmen und fordern stattdessen, dass „competence should be regarded as a process, a continuum with many steps in between" (2015, S. 7). Die Skalierung kompetenten Wissens und Könnens inklusive Messung erreichter (bzw. Angabe noch zu erreichender) Level wird dadurch aber bestimmt nicht vereinfacht!

Was im Rahmen von international ausgerichteter Forschung und Schulleistungsstudien mit Dominanz der Entwicklung und Erprobung von Testverfahren zur Beschreibung und Beurteilung der Kompetenzentwicklung mit entsprechenden personalen und sachlichen Ressourcen einschließlich komplexen statistischen Analyseinstrumenten an Kompetenzmessung einigermaßen stringent auf- und nachgewiesen werden kann, stellt sich für den Schulhaus-Gebrauch als schier unüberwindbare Hürde dar. Soll es eben nicht nur um die Feststellung des Status, des Ausmaßes und der erreichten Höhe in der Verfügung von (Fakten-)Wissen gehen, 


\section{Lernstand - Lernwirksamkeit - Lernleistung}

sondern gerade um das erreichte Niveau kompetenter „Selbst- und Weltreferenz“, sind Lehrkräfte wohl heillos überfordert - und zwar bereits aus prinzipiellen Erwägungen bezüglich der Qualität der Lehrerurteile und jenseits der Problematik mit den „Risiken und Nebenwirkungen“ der Zensuren (vgl. Breidenstein, 2015)!

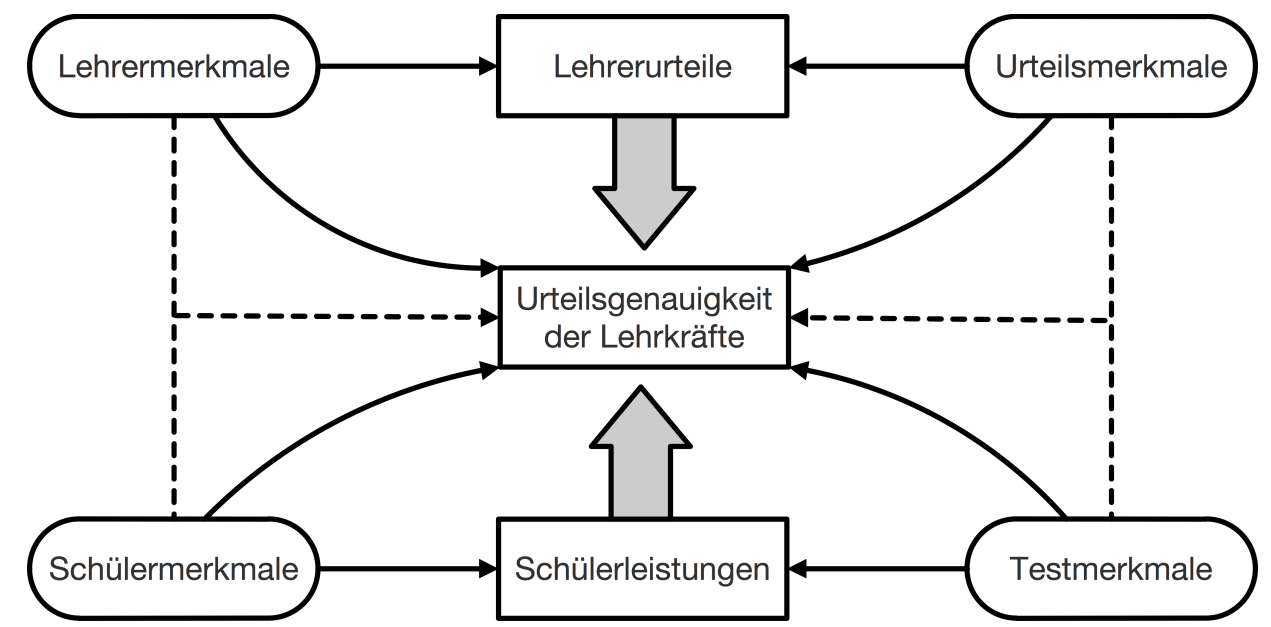

Abb. 1: Heuristisches Modell der Moderatoren der Urteilsgenauigkeit von Lehrkräften (Quelle: Eigene Darstellung nach Südkamp, Kaiser \& Möller, 2014, S. 6)

Wie „akkurat“ Lehrerurteile überhaupt sein können (Artelt \& Rausch, 2014; Rausch, Matthäi \& Artelt, 2015), hängt nämlich von vielen und unterschiedlichen Faktoren ab. Südkamp, Kaiser und Möller $(2012 ; 2014)$ haben dazu ein heuristisches Modell vorgestellt, das die Moderatoren der Urteilsgenauigkeit von Lehrkräften systematisiert. Demnach ergibt sich die Urteilsgenauigkeit aus dem Ausmaß der Übereinstimmung von Schülerleistung und Lehrerurteil, wobei das Urteil der Lehrkräfte von Lehrermerkmalen wie Berufserfahrung, Professionswissen etc. und der Art der Beurteilung (Zeugnisart, Beurteilungsstufen etc.) abhängt. Schülermerkmale, wie Vorwissen, Motivation etc. und die Testmerkmale (Fach, Art etc.) wiederum beeinflussen die zu beurteilenden Schülerleistungen. Die Merkmalsbereiche Lehrer-, Schüler-, Urteils- und Testmerkmale inklusive der Übereinstimmung zwischen Urteils- und Testmerkmalen und zwischen Lehrer- und Schülermerkmalen bestimmen somit die „Akkuratesse“ der Lehrerurteile.

\subsection{Input - Outcome}

Mit den im Kontext von Lernen und Bildung als erfolgreich konstatierbaren Veränderungen - einer konstruktivistischen Grundlegung lerntheoretischer Konzeptionen und einer kompetenzorientierten Ausrichtung der Lernprozesse - ist ja nicht das 


\section{Lernstand - Lernwirksamkeit - Lernleistung |}

„klassische Handwerkszeug“ des Lehrberufs obsolet geworden, sind Unterrichts- und Sozialformen, die Lehrverfahren, sonstige didaktisch-methodische Maßnahmen, der Medieneinsatz etc. nicht umgekrempelt worden - warum und wie auch? Vielmehr hat sich in der Tat die Denkrichtung radikal geändert: Üblicherweise in den Kategorien „lehrseits-lernseits“ (Schratz, 2009; Eckart \& Schratz, 2015) denkend, ergibt sich eine jeweils pointierte/re Sicht auf die ,Antipoden':

Bei einer lehrseitigen Orientierung von Unterricht liegt der Fokus der Wahrnehmung und Wirkmacht bei der Lehrperson während eine Orientierung, lernseits' von Unterricht diesen auf die Seite der Lernenden verschiebt. ,Lernseits' beleuchtet demnach das Unterrichtsgeschehen auf Seite der Schülerinnen und Schüler. (Eckart \& Schratz, 2015, S. 194)

Im wohlverstandenen Sinne wird dann der Input an personalen und sachlichen Ressourcen für das Lehren und Lernen durchaus professionell konzipiert und operationalisiert, um als Output die von den erwarteten mehr oder weniger abweichenden Ergebnissen, auch defizitäre Wirkungen zu diagnostizieren und als formale Zuweisung für Abschlüsse, darauf für aufbauende Bildungsgänge oder Berufseinstiege zu qualifizieren.
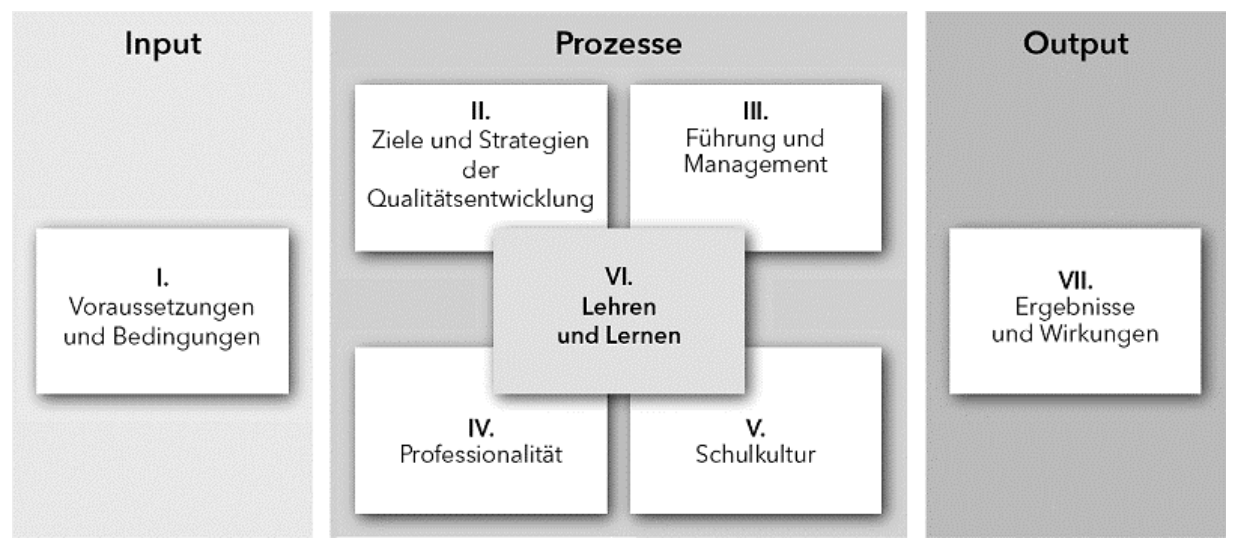

Abb. 2: Lehren und Lernen als Qualitätsbereich schulischer Entwicklung (Quelle: Institut für Qualitätsentwicklung, 2011, S. 4)

Mit dem Fokus auf Kompetenzen als gewünschtem nachhaltigen Outcome wird von diesem her sowohl der Input an personalen und sachlichen Ressourcen bestimmt als auch der in entsprechenden Strukturen und Prozessen von Lehr-Lern-Szenarien organisierte Throughput gestaltet; als mess- und beurteilbarer kurzfristiger Output ist die formale Qualifizierung und Positionierung darin durchaus inkludierbar!

Für die Bilanzierung der Ergebnisse und Wirkungen einer kompetenzorientierten Konzeption von Lernen ist eine durchgängige „Musterung“ der gesamten „Wertschöpfungskette" vom Outcome her unerlässlich und dementsprechend komplex: Insoweit die gewünschten bzw. für notwendig erachteten Kompetenzen begründet 


\section{Lernstand - Lernwirksamkeit - Lernleistung}

und formuliert sind, gilt es zu klären, über welche Voraussetzungen die Lernenden in inhaltlich-fachlicher, aber auch lernstrategischer Hinsicht verfügen, was deren Status im Hinblick auf die Kompetenzentwicklung an Potenzial erwarten lässt und welche Restriktionen zusätzliche didaktisch-methodische Anstrengung benötigen.

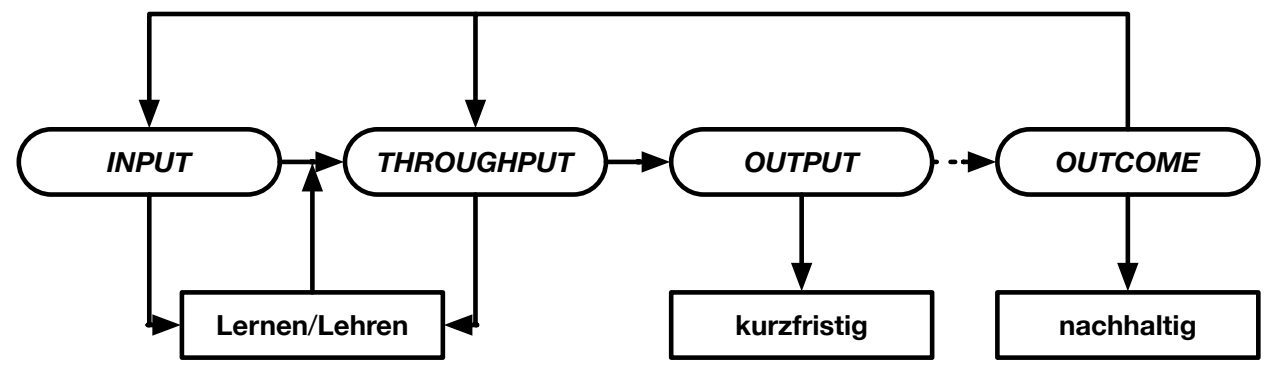

Abb. 3: Von Outcome zum Input - und wieder zurück! (Quelle: Eigene Abbildung)

Für das Lernen und Lehren sind zu treffende Maßnahmen konzeptionell, strukturell und prozessual so abzustimmen, ob sie das Mehr an Kompetenz in der Entwicklung ermöglichen und deshalb auch kontinuierlich zu überprüfen sind, inwieweit der erreichte Lernstand dem weiteren Aus- und Aufbau der Kompetenzen förderlich bzw. gegebenenfalls neu zu justieren ist.

Insofern mit Unterricht und Erziehung nach wie vor auch eine gesellschaftlich relevante Zuweisung (Stichwort Leistungsgesellschaft) stattfindet und individuelle Teilhabechancen reguliert werden, bleibt die damit verbundene Feststellung von Qualifikation und damit einhergehend die Positionierung im Gesellschaftsgefüge nach wie vor Aufgabenstellung des Bildungssystems - Kompetenzorientierung hin oder her!

Mit Kompetenzen als nachhaltigem Outcome wird allerdings nicht allein die disponible Verfügung über fachspezifisches Wissen postuliert, sondern mit dem Fachwissen sollen in der Gestaltung von Lernumgebungen gleichzeitig und gemeinsam multiple und mehrdimensionale Ziele (vgl. Renkl, 2016) verfolgt und auch erreicht bzw. dazu fach-/fächerübergreifende Kompetenzen entwickelt und gefördert, quasi „zwei Fliegen mit einer Klappe“ (Wecker, Hetmanek \& Fischer, 2016) geschlagen werden. In Bezug auf die Rolle fächerübergreifender Kompetenzen für den Erwerb von Fachwissen gilt es allerdings zwei Konstellationen zu unterscheiden:

- Als neutral anzusehen sind unterrichtliche Maßnahmen, bei denen ,zwar ein positiver Effekt auf den Erwerb der fächerübergreifenden Kompetenz auftritt, jedoch kein positiver Effekt auf den Erwerb von Fachwissen“" (Wecker, Hetmanek \& Fischer, 2016, S. 229) zu erkennen ist: z. B. „Handhabungskompetenz von Hard- und Software“ (ebd., S. 230) beim Einsatz von IKT-Technik im Unterricht. 


\section{Lernstand - Lernwirksamkeit - Lernleistung |}

- Als funktional erweisen sich hingegen Maßnahmen, denen in „der Förderung einer fächerübergreifenden Kompetenz ein positiver Beitrag zum Lernerfolg in Bezug auf das Fachwissen“ (Wecker, Hetmanek \& Fischer, 2016, S. 229) zu bescheinigen ist: z. B. Einsatz von Lern- und Argumentationstrategien und Concept Maps.

In der Formulierung von kompetenzbasierten und -orientierten Fähigkeiten, Fertigkeiten und Bereitschaften wird zudem immer sehr deutlich die Verklammerung von Wissen und Handeln - und zwar für und in der Lebenswelt der Lernenden - thematisiert. Die Überprüfung der getroffenen Maßnahmen hinsichtlich ihrer Lernwirksamkeit ist deshalb auch nicht trivial:

Der angemessenen Erfassung von Kompetenz kommt eine Schlüsselfunktion zu. Die Herausforderung für Forschende (und Praktiker W. B.) ist dabei, dass verschiedene Erfassungsmethoden jeweils unterschiedliche Gültigkeitsbereiche für sich beanspruchen können. (Kunter \& Klusmann, 2010, S. 76)

Zur Kompetenzerfassung stehen unterschiedliche Zugänge zur Verfügung: Mit einer subjektiven Erfassung nimmt das „Individuum selbst eine Einschätzung seiner Kompetenzen vor, etwa durch Bewertung des eigenen Lernerfolgs in der Aus- und Weiterbildung“, mittels einer objektiven Erfassung „erfolgt die Erhebung ,von außen“ anhand von externen Kriterien“ und einer ,distalen und der proximalen Erfassung des Zielkonstrukts“ (Kunter \& Klusmann, 2010, S. 75). Distale Indikatoren stellen z. B. klassische Leistungstests, Punkte- und Notenvergabe dar, proximale Indikatoren zielen z. B. auf die Erfassung kognitiver Merkmale und Lernstrategien durch spezifische Wissenstest bzw. Fragebögen.

\subsection{Effekt - Qualität}

Es ist ja nicht ungewöhnlich, wenn mit der Frage „What works best in school“ (Köller, 2012) registriert wird, dass Lernprozesse bessere oder weniger gute Qualität aufweisen können - diese ist schließlich ,abhängig von individuellen Voraussetzungen, aber natürlich auch von den Voraussetzungen der Lernumgebung. Ziel des Unterrichts ist daher die Unterstützung von nachhaltigen Lernprozessen durch eine Förderung des aktiven Aufbaus von Wissen“ (Kunter \& Ewald, 2016, S. 19).

$\mathrm{Ob}$ John Hattie mit seiner akribischen Meta-Analyse tatsächlich den „Heiligen Gral“ (Terhart, 2011) gefunden, endlich „das Lernen sichtbar“ und ausfindig gemacht hat, was guten Unterricht ausmacht, lässt sich wohl nicht in toto belegen; hingegen ist erstmalig in diesem Umfang dokumentiert worden, welche Faktoren erfolgreiches Lernen bedingen und ermöglichen können.

Klaus Zierer hat die von Hattie aggregierten Daten nochmals geclustert und kann für den Einfluss auf den Lernerfolg feststellen, dass knapp 40\% auf die Lehrperson 


\section{Lernstand - Lernwirksamkeit - Lernleistung}

und die unterrichtliche Lehre entfallen - zusammen mit curricularen Lehrplänen summiert sich dies auf fast $60 \%$ (Zierer, 2016)!

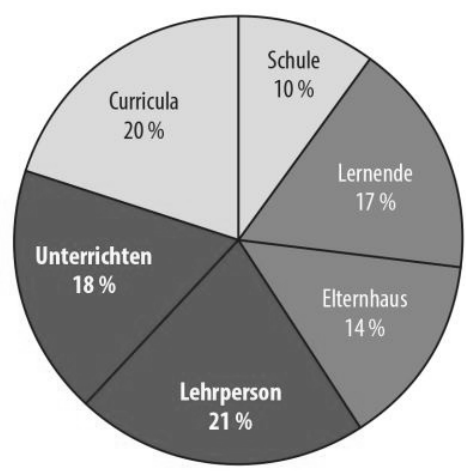

Abb. 4: Einflüsse auf den Lernerfolg (Quelle: Zierer, 2016, S. 87)

Daraus kann man sicherlich für „gestresste Lehrer“ (Zierer, 2016) herauslesen, dass „Lehrkräfte nicht ausschließlich als Randbedingung für unterschiedliche Lernerfolge von Schülerinnen und Schülern zu sehen“ (Kunter, Seidel \& Artelt, 2015, S. 59) sind, es doch sehr auf sie ankomme - aber so ungewöhnlich ist dies auch wieder nicht: Selbst wenn das Lernen - wenig verwunderlich - die Domäne der Lernenden ist, wird mit den Befunden aber auch deutlich, dass der Effekt des Unterrichts, die Professionalität der Lehrkräfte und die Qualität der Curricula doch konstitutiv und damit zentral sind - und lediglich radikale Verfechter eines ebenso radikal offenen Unterrichts irritieren dürfte!

Dass die Qualität des Unterrichts mit einer Effektstärke ${ }^{4}$ von $\mathrm{d}=0.44$ (vgl. Abb. 5) nicht nur in den Bereich der ,gewünschten Effekte“ fällt, ist als erfreulich festzustellen, sondern auch der Verweis darauf, dass Unterrichtsqualität mehr als der sonst gerne kolportierte ,gute Unterricht“، ist.

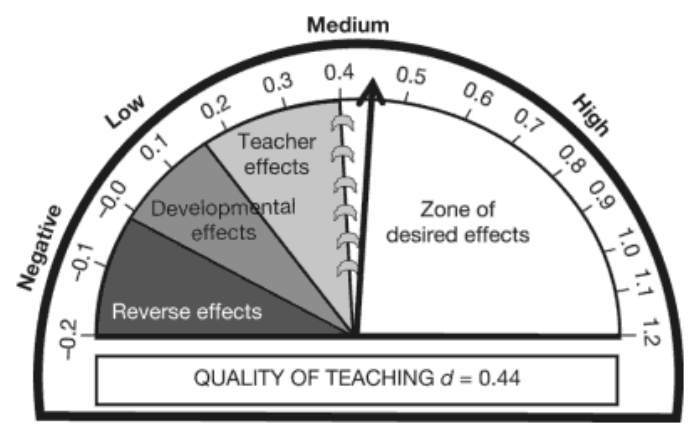

Abb. 5: Gewünschter Effekt: Quality of teaching (Quelle: Hattie, 2009, S. 115) 


\section{Lernstand - Lernwirksamkeit - Lernleistung |}

Nach Kunter und Ewald (2016, S. 10) und mit Bezug auf Berliner (2005) ergibt sich „qualitätsvoller Unterricht“ quasi nach der einfachen Formel guter Unterricht + effektiver Unterricht, wobei mit gutem Unterricht die Einhaltung normativer Prinzipien und mit effektivem Unterricht das tatsächliche Erreichen der Lernziele gemeint ist und als qualitätsvoller Unterricht ,beide Prinzipien vereint, d. h. auf Unterrichtsformen, die unseren (pädagogischen) Wertvorstellungen entsprechen und gleichzeitig die Lernziele erreichen“ (Kunter \& Ewald, 2016, S. 12).

In der empirischen Bildungsforschung (kritische Einwendungen dazu bei Gruschka, 2007) werden als essenzielle Dimensionen der Unterrichtsqualität insbesondere die Klassenführung (in ähnlicher Diktion etwas deutlicher als ClassroomManagement bezeichnet), die kognitive Aktivierung und konstruktive Unterstützung (vgl. Holzberger \& Kunter, 2016, S. 43-45) betrachtet: Sie ,stellen eine sparsame, aber relativ umfassende Systematisierung der im Unterricht zu beobachtenden LehrLernprozesse dar und sie haben sich im deutschen Sprachraum sehr gut etabliert, nicht zuletzt auch daher, weil mittlerweile gute Instrumente für deren Erfassung vorliegen“" (Kunter \& Ewald, 2016, S. 16):

- Klassenführung: „Hier geht es um die Steuerung des Unterrichts, sodass möglichst wenige Störungen auftreten, alle Schüler/innen beteiligt sind und die Unterrichtszeit effektiv genutzt wird“" (Kunter \& Ewald, 2016, S. 15).

- kognitive Aktivierung: „Damit ist gemeint, inwieweit die Lernenden angeregt werden, sich vertieft mit dem Lernstoff auseinanderzusetzen, das heißt also, inwieweit der Unterricht so angelegt ist, dass er hochwertige kognitive Prozesse auslöst, von denen angenommen wird, dass sie zu nachhaltigem Lernen führen" (Kunter \& Ewald, 2016, S. 15/16).

- konstruktive Unterstützung: „In dieser dritten Dimension stehen die Unterstützung der Lernenden bei Verständnisproblemen und die Schaffung eines Lernklimas, in dem die Interaktion zwischen Lehrkräften und Lernenden von Respekt und Wertschätzung geprägt ist, im Mittelpunkt“" (Kunter \& Ewald, 2016, S. 16).

\section{Angebot - Nutzung}

Das Angebots-Nutzungs-Modell ist „ein in der schulischen Unterrichtsforschung verbreitetes Rahmenmodell, das die komplexe Wirkungsweise des Unterrichts veranschaulichen soll. Das (...) Modell basiert auf Grundgedanken von H. Fend und wurde von den Autoren gemeinsam mit F. E. Weinert weiterentwickelt“ (Helmke \& Schrader, 2016).

Was zunächst ökonomistische Assoziationen wecken könnte, nämlich Lernen, Unterricht, Erziehung, Bildung (inkl. Kompetenzen) und letztendlich Selbst- und 


\section{Lernstand - Lernwirksamkeit - Lernleistung}

Weltsicht einem Prozess der Aushandlung und gegenseitigen Verpflichtung zur Erbringung von Leistungen auszuliefern, bildet jedoch ab, was in lerntheoretischer Sicht auf die Lernenden und mit professionstheoretischem Blick auf die Lehrenden Sinn macht.

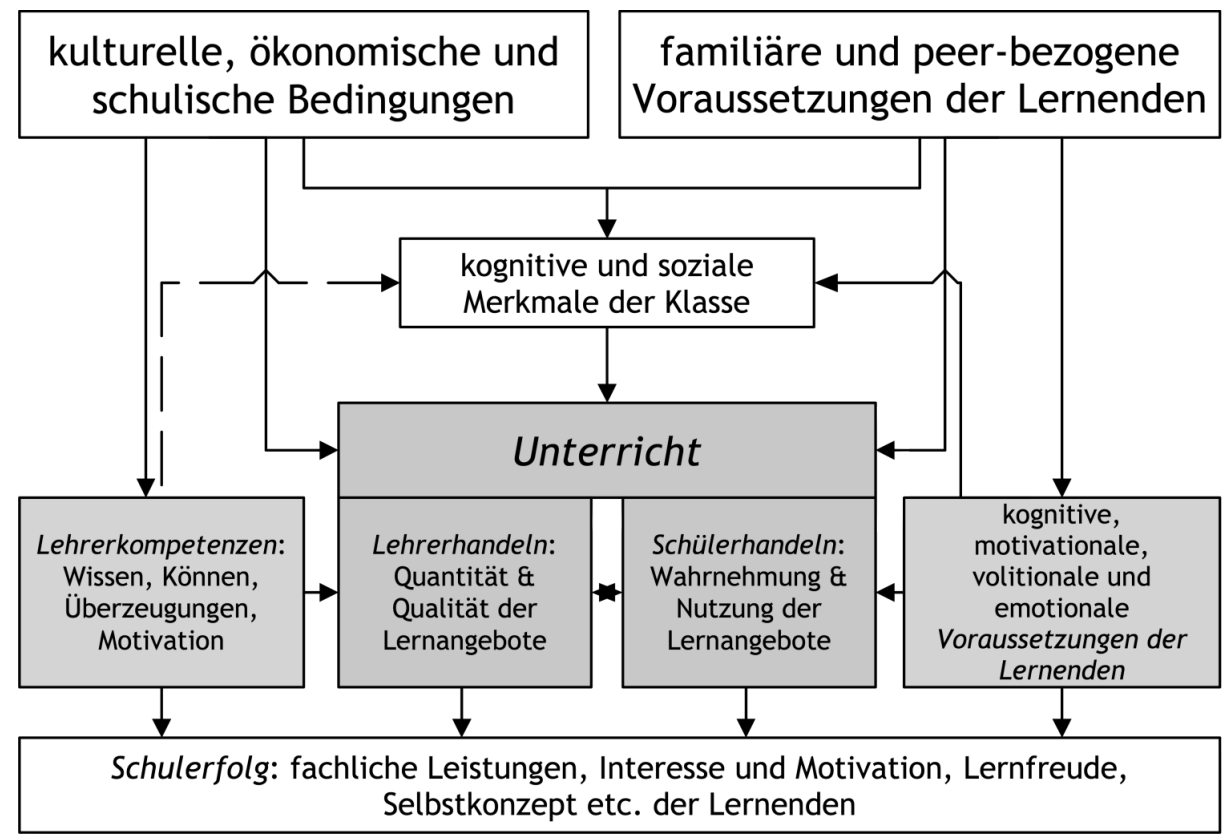

Abb. 6: Vereinfachtes Angebots-Nutzungs-Modell (verändert aus: Lipowsky, 2006, S. 48)

Lernen als recht eigensinnige und eigenwillige Konstruktion von Bedeutung, lässt sich von Seiten der Lehrenden nicht erzwingen und kann von diesen in der Tat nur als Lernangebot in entsprechenden Lernumgebungen konzipiert und Lernsituationen operationalisiert werden - allerdings mit bestmöglichen Ressourcen an Wissen, Können, Überzeugung und Motivation, allergrößter Sorgfalt an die Lernenden herangetragen und auf deren kognitive, motivationale, volitionale, emotionale und soziale Voraussetzungen abgestimmt (vgl. Abb. 6):

Dabei ist die Klärung der Rollen von Lehrenden und Lernenden von großer Bedeutung. So liegt die Verantwortung für das Lernen bei den Schülerinnen und Schülern. Sie muss ihnen zugemutet und auch zugetraut werden. Die Lehrkraft hat die Verantwortung für das Lehren. Ihre Aufgabe ist es, Lernarrangements so zu gestalten, dass alle Schülerinnen und Schüler die Unterrichtsangebote nutzen können, sich auf ihrem individuellen Lernstand angesprochen fühlen und die notwendigen Hilfen bekommen. Damit Lernen für alle Schülerinnen und Schüler erfolgreich verläuft, spielen individuelle Förderung und die Stärkung des eigenverantwortlichen Lernens durch 


\section{Lernstand - Lernwirksamkeit - Lernleistung |}

entsprechende Unterrichtsarrangements eine wichtige Rolle. (Institut für Qualitätsentwicklung, 2011, S. 23)

\subsection{Struktur - Prozess}

Guter Unterricht entsteht in den Tiefenstrukturen.

(Kunter \& Trautwein, 2013, S. 105)

„Methodenwochen sind Zeitverschwendung“ schreiben fast schon apodiktisch Felten und Stern (2012, S. 144) und deuten damit auf ein Phänomen, das sich in der Betrachtung von Unterricht allerdings zwangsläufig ergibt: Vieles an Aktivität von Lehrenden und Lernenden ist auch für Außenstehende deutlich erkennbar: die Organisation des Unterrichts, der Einsatz der Sozialformen, die Methodenvariation etc.

Diese sind als Bedingungen für den Lernerfolg von den Lehrkräften nach professionellen Gesichtspunkten in die Gestaltung der Lernumgebungen einzubringen und dementsprechend als deren sichtbare Struktur kenntlich zu machen - aber auch nicht zu verwechseln mit dem eigentlichen Vorgang des Lernens.

Zentral in diesem Zusammenhang ist die Unterscheidung von „Wirksamkeit“ und „Wirkungsweise“. Analysen im Rahmen des Prozess-Produkt-Paradigmas erlauben Aussagen über die Wirksamkeit einzelner Faktoren oder bestimmter Faktorstrukturen, aber sie vermögen nicht die Wirkungsweise unterrichtlicher Mittel in Lehr-LernInteraktion zu erklären. (Minnameier, Hermkes \& Mach, 2015, S. 838)

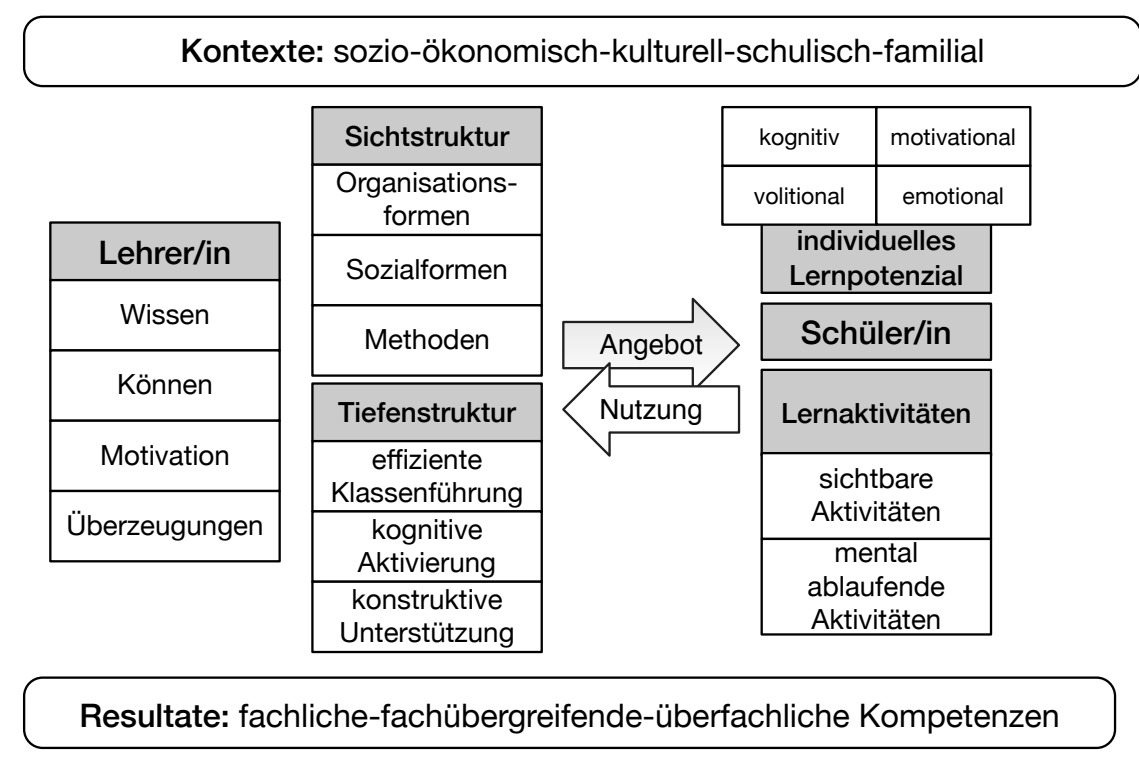

Abb. 7: Kontexte - Sichtstruktur - Tiefenstruktur - Resultate (Quelle: Eigene Darstellung) 


\section{Lernstand - Lernwirksamkeit - Lernleistung}

„Sichtstrukturen stecken den Rahmen für die Tiefenstrukturen ab“ (Kunter \& Ewald, 2016, S. 14), d. h. die für das Lernen konstitutiv mental ablaufenden Aktivitäten sind ihrer Natur nach das Entscheidende, aber als solche nicht unmittelbar sichtbar, aber als ,,Tiefenstruktur von Unterricht in den Fokus zu nehmen und Unterrichtsqualität gerade auch auf Lehr-Lern-Prozessebene sichtbar zu machen, insbesondere unter dem Aspekt verständnisvollen Lernens“ (Minnameier, Hermkes \& Mach, 2015, S. 840). Nach Kunter und Trautwein (2013), ,ist empirisch vielfach belegt, dass die Tiefenstrukturen des Unterrichts das Lernen und die Entwicklung von Schülerinnen und Schülern deutlich stärker beeinflussen als die Sichtstrukturen, also die Organisationsformen oder Methoden“ (S. 76). In und mit den Tiefenstrukturen findet der eigentliche Lehr-Lernprozess statt: „Es geht um den Austausch (die Interaktionen) zwischen Lernenden und Lehrenden und um die Beschäftigung der Lernenden mit dem Lern-inhalt"، (Kunter \& Trautwein, 2013, S. 76) und der Frage- und Problemstellung

wie Denkprozesse der Lernenden in Gang gesetzt und unterstützt werden und wie insbesondere auch auf Denkfehler und Fehlkonzeptionen eingegangen wird. LehrLern-Prozessforschung erfordert insofern auch stets eine fachdidaktische Fundierung, im Rahmen derer die Struktur von Inhalten und Kompetenzen herausgearbeitet wird. (Minnameier, Hermkes, \& Mach, 2015, S. 840)

Als exponierte Dimensionen zur Beschreibung der Tiefenstrukturen des Unterrichts in empirischen Untersuchungen gelten die Klassenführung, kognitive Aktivierung und konstruktive Unterstützung (siehe Kap. 2.2).

Die Gelegenheit zur inhaltlichen Auseinandersetzung setzt ein ausreichendes Maß an zur Verfügung stehender Lernzeit, die aktive und intensive Nutzung dieser Lernzeit (time on task), eine effektive Klassenführung als Voraussetzung für eine intensive Lernzeitnutzung, die Nutzung der zur Verfügung stehenden Lernzeit für inhaltlich relevante Aspekte des Unterrichtsgegenstands sowie eine klare und gut strukturierte Unterrichtsgestaltung voraus. (Lipowsky, 2006, S. 56)

\subsection{Außensteuerung - Innensteuerung}

Mit dem Blick auf Sichtstrukturen und Tiefenstrukturen des Unterrichts und dem Konzept der kognitiven Aktivierung (vgl. Heymann, 2015) als „Prozess der Induktion eines Problems beim Lernenden“ und der konstruktiven Unterstützung als „Anleitung und Begleitung des dadurch in Gang gesetzten Problemlöseprozesses“ (Minnameier, Hermkes \& Mach, 2015, S. 842) ändert sich auch die Perspektive auf die Prozesse der Außensteuerung und Innensteuerung des Lernens.

Wenn auch aus ,pragmatischen Gründen“ (Prediger \& Scherres, 2012) das Niveau eines Lernprozesses oftmals schlicht an seinem Ergebnis gemessen wird - Wie vollständig und fachlich korrekt haben die Lernenden eine Aufgabenstellung bewältigt? - bleibt die Frage nach der kognitiven Aktivierung, der Qualität der inhaltlichen 


\section{Lernstand - Lernwirksamkeit - Lernleistung |}

Auseinandersetzung, der inhaltlichen Strukturierung, der Instruktionsqualität und Einbettung situierten Lernens in konstruktivistische Lernumgebungen auf der Tagesordnung und insofern rückt insbesondere die Frage nach der Qualität der Aufgaben selbst und damit auch die Arbeit an und mit Begriffen, Konzepten und Argumenten als unerlässliche Bausteine für mentale Konstruktionsprozesse im kompetenzorientierten Unterricht (vgl. Brandl, 2012) in den Fokus.

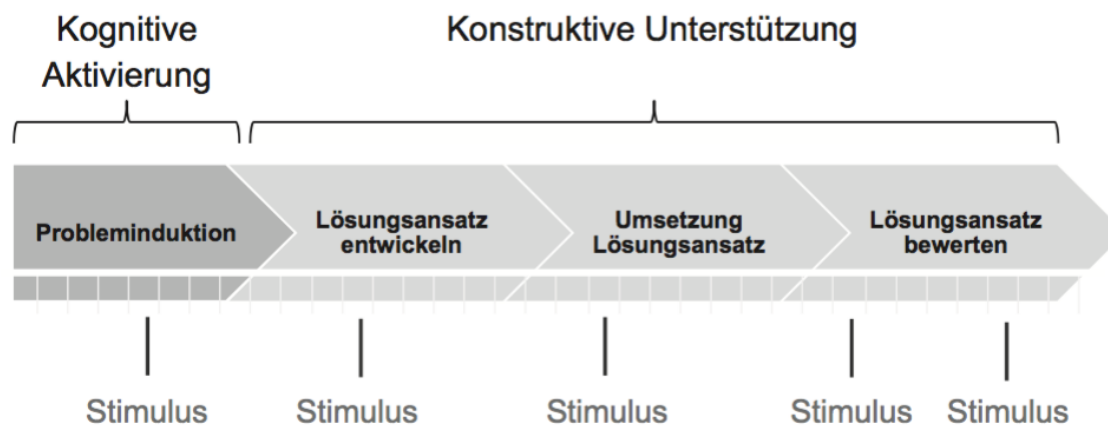

Abb. 8: Aufgabenzyklus und Binnenstimuli (Quelle: Minnameier, Hermkes \& Mach, 2015, S. 849)

Die Beantwortung der Fragen, warum welche Aufgaben dafür (besser) geeignet sind, welche Kriterien für ihre Formulierung maßgebend sind, welches Format sie besitzen sollen, in welchen Kontext sie eingebettet werden können, welches Abstraktionsniveau sie repräsentieren müssen etc. ist für die Qualität der kognitiven Aktivierung essenziell. Mit der Entwicklung eines ,allgemeindidaktischen Kategoriensystems ${ }^{\text {“5 }}$ (vgl. Tab 1) soll es möglich sein, die „Spreu vom Weizen“ zu trennen:

Das Aufgabenanalysesystem stellt kein umfassendes didaktisches Planungs- und Analysemodell dar, sondern konzentriert sich auf Fragen der kognitiv-aktivierenden Ausgestaltung von Aufgaben. Es soll so ausgelegt sein, dass unterschiedliche Aufgabenarten auch von Lehrkräften, die das Fach fachfremd unterrichten, eingeschätzt werden können. (Kleinknecht et al., 2011, S. 333)

Tab. 1: Allgemeindidaktisches Kategoriensystem zur Aufgabenanalyse (Quelle: Kompilation aus Maier et al., 2010, S. 90; Bohl et al., 2015, S. 29)

\begin{tabular}{l|c}
\hline Kategorie & Subkategorie \\
\hline Wissensart & Fakten Prozeduren $\quad$ Konzepte metakognitives Wissen \\
\hline $\begin{array}{l}\text { kognitiver } \\
\text { Prozess }\end{array}$ & Reproduktion nah $\leftarrow$ Transfer $\rightarrow$ weit kreatives Problemlösen \\
\hline
\end{tabular}


Lernstand - Lernwirksamkeit - Lernleistung

\begin{tabular}{l|c|c|c}
\hline $\begin{array}{l}\text { Wissens- } \\
\text { einheiten (WE) }\end{array}$ & $1 \mathrm{WE}$ & 2 bis 4 WE & \multicolumn{1}{c}{$>4 \mathrm{WE}$} \\
\hline Offenheit & $\begin{array}{c}\text { definiert/ } \\
\text { konvergent }\end{array}$ & $\begin{array}{c}\text { definiert/ } \\
\text { divergent }\end{array}$ & $\begin{array}{c}\text { schlecht definiert/ } \\
\text { divergent }\end{array}$ \\
\hline $\begin{array}{l}\text { Lebenswelt- } \\
\text { bezug }\end{array}$ & ohne konstruiert konstruiert/authentisch real \\
\hline $\begin{array}{l}\text { sprachlogische } \\
\text { Komplexität }\end{array}$ & einfach \\
\hline $\begin{array}{l}\text { Repräsentations- } \\
\text { formen }\end{array}$ & 1 Repräsentationsform Integration Integration/Transformation \\
\hline
\end{tabular}

Im Lernfeld Ernährung, Haushalt und Konsum nimmt der Lebensweltbezug eine prominente Stelle ein und kommt so eigentlich ohne die ,traditionell didaktische Diskussion zur Anbindung schulischer Themen und Aufgaben an die Alltags- und Lebenswelt der Schülerinnen und Schüler" (Kleinknecht et al., 2011, S. 335) aus. Nichtsdestotrotz sind gerade hinsichtlich der Relationierung domänenspezifischen Fachwissens und der Erfahrungs- und Lebenswelt der Schülerinnen und Schüler besondere Beachtung und Sorgfalt in der Aufgabenstellung angesagt:

Maier et al. (2010, S. 89) gelangen diesbezüglich zu vier unterschiedlichen Ausprägungen von Aufgabenstellungen, nämlich solche

- ohne Lebensweltbezug: es wird keine Verknüpfung zwischen Fachwissen und der Erfahrungs- und Lebenswelt der Schülerinnen und Schüler vorgegeben oder gefordert.

- mit konstruiertem Lebensweltbezug: es wird eine Verknüpfung zwischen Fachwissen und einer stark konstruierten Lebenswelt vorgegeben oder gefordert (die aber eher nicht den Erfahrungen der Lernenden entspricht; Analogien zur eigenen Erfahrung sind kaum erkennbar).

- mit konstruiertem, aber authentisch wirkendem Lebensweltbezug: es wird ein Lebensweltbezug konstruiert, der im Zusammenhang der Aufgabenstellung Sinn macht und authentisch wirkt.

- mit realem Lebensweltbezug: die Differenz zwischen Aufgabe und der Erfahrungs- und Lebenswelt der Lernenden tendiert insofern gegen Null, als die Problemstellung lebensweltlich relevant ist und auch tatsächlich gelöst werden soll. 


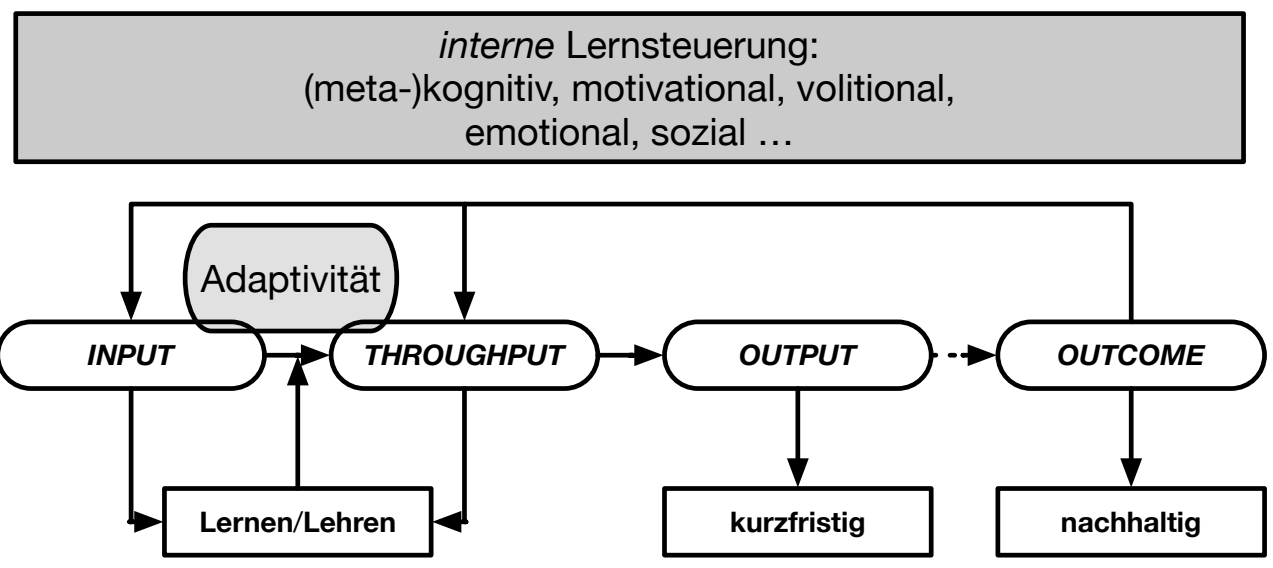

externe Lernsteuerung:

Unterrichtsqualität, Aufgabenstellung, Motivation, Methodenvariation, Feedback ...

Abb. 9: Adaptive Außensteuerung - Innensteuerung (Quelle: Eigene Abbildung)

Man muss zwar nicht nach völlig „neuen Lernformen“ suchen, um ,ein zunehmend selbstbestimmtes, selbstverantwortliches und selbstorganisiertes Lernen der Lernenden“ (Kipmann, 2015, S. 82) zu ermöglichen, dennoch sind Lehre und Lernen so aufeinander abzustimmen, dass der nachhaltige Outcome auch gewährleistet werden kann. Zum Erreichen solch erwünschter Arbeitsergebnisse ist der Blick auf die Adaptivität als Prozessmerkmal und damit verbundener adaptiver Lehrkompetenz (Beck et al., 2008) als Professionsmerkmal zu richten, da „die Lernwirksamkeit von Unterrichtsaktivitäten stärker durch die Prozessintensität als durch die Richtigkeit des Ergebnisses bestimmt wird“ (Prediger \& Scherres, 2012).

Die Abstimmung externer Lernsteuerung (Qualität des Unterrichts, Aufgabenstellung, Motivation, Methodenvariation, Feedback etc.) durch die Lehrenden auf die kognitiv, motivational, volitional, emotional, sozial etc. interne Lernsteuerung der Lernenden ist grundlegende Bedingung für das Gelingen und erfordert nach (Geier, 215, S. 10)

- das Herstellen eines Sinnbezugs und Bedeutungszusammenhangs

- die Förderung individueller Selbstständigkeit

- die Autonomie der Lernenden

- ein problemlösendes forschendes Lernen

- $\quad$ eine Förderung und Freisetzung kreativer Lösungsansätze

- das Anbieten differenzierter Zugänge. 


\section{Lernstand - Lernwirksamkeit - Lernleistung}

\section{Ausblick}

Lernwirksam unterrichten bedeutet nach Felten und Stern (2012, S. 144), dass ,erfolgreiche Lehrpersonen“" z. B. wissen, dass

- Kinder etwas lernen und leisten wollen,

- viele Wege zum Unterrichtserfolg führen,

- das Vorwissen schon eine erhebliche Rolle spielt,

- es sehr auf die Qualität der Aufgaben ankommt und diese besonders motivierend und lernförderlich sind, wenn

- $\quad$ sie kognitiv herausfordernd und trotzdem zu bewältigen sind,

- sie unterschiedliche Lösungen auf verschiedenen Niveaus zulassen,

- die Kontexte der Aufgaben den Lernenden nachvollziehbar erscheinen.

Und sie können vor allem

- Lernsequenzen zielorientiert strukturieren,

- Lernprozesse abwechslungsreich moderieren,

- auf vielfältige Art und Weise verbalisieren und visualisieren,

- flexibel erklären und variabel argumentieren,

- Wissen stringent strukturieren und spannend präsentieren sowie

- angemessen Hilfestellung geben.

Dieses umfangreiche Programm in adäquater Weise umzusetzen, ist schon Aufgabenstellung genug. Den damit intendierten Lernerfolg als Lernstand zu dokumentieren, als Lernwirksamkeit zu qualifizieren und als Lernleistung zu quantifizieren, ist und bleibt eine Problemstellung par excellence!

\section{Anmerkungen}

1 Was die entscheidenden Voraussetzungen jedweden Lernens anbelangt - Sinneswahrnehmung und Denken - hat sich in den vielfältigen lern- und erkenntnistheoretischen Positionen seit den Tagen Leibniz', Kants und Hegels eigentlich prinzipiell wenig geändert: „Selbstverständlich ist nichts im Verstande, was nicht zuvor in den Sinnen war - mit Ausnahme des Verstandes selbst“" (Leibniz, 1669, AA VI, 2, S. 393). „Anschauung und Begriffe machen also die Elemente aller unserer Erkenntnis aus, so daß weder Begriffe, ohne ihnen auf einige Art korrespondierende Anschauung, noch Anschauung ohne Begriffe, ein Erkenntnis abgeben können. (...) Gedanken ohne Inhalt sind leer, Anschauungen ohne Begriffe sind blind. Daher ist es ebenso notwendig, seine Begriffe sinnlich zu machen, (d. i. ihnen den Gegenstand in der Anschauung beizufügen) als seine 


\section{Lernstand - Lernwirksamkeit - Lernleistung |}

Anschauungen sich verständlich zu machen (d. i. sie unter Begriffe zu bringen). Beide Vermögen, oder Fähigkeiten, können auch ihre Funktionen nicht vertauschen. Der Verstand vermag nichts anzuschauen, und die Sinne nichts zu denken. Nur daraus, daß sie sich vereinigen, kann Erkenntnis entspringen“" (Kant, 1781, Kritik der reinen Vernunft, Zweiter Teil, Die Transzendentale Logik). „Abstrakt lernt man denken durch abstraktes Denken. Man kann nämlich entweder vom Sinnlichen, Konkreten anfangen wollen und dieses zum Abstrakten durch Analyse heraus- und hinaufpräparieren, so - wie es scheint - den naturgemäßen Gang nehmen, wie auch so vom Leichteren zum Schwereren aufsteigen. Oder aber man kann gleich vom Abstrakten selbst beginnen und dasselbe an und für sich nehmen, lehren und verständlich machen (Hegel, 1835, Werke Bd. XVII, S. 400).

2 Nach Heckhausen kann man dann von Leistung im pädagogischpsychologischen Sinne sprechen, wenn

1. ein Handlungsergebnis vorliegt,

2. das Handlungsresultat einem Urheber zugeschrieben werden kann, der das Ergebnis auch beabsichtigt hat,

3. die Handlungen in ihren Ergebnissen gelingen oder misslingen können,

4. das Handlungsergebnis auf einen Schwierigkeitsmaßstab und/oder auf einen Maßstab für die benötigte Anstrengung beziehbar ist,

5. dem Schwierigkeitsmaßstab ein (z. B. sachbezogener, personbezogener, sozialbezogener) Gütemaßstab entspricht, den man zur Beurteilung der Handlungsergebnisse für verbindlich hält (vgl. Brunner \& Zeltner, 1980, S. 131).

3 Im Kontext der „Kompetenzorientierung im Unterricht aus der Perspektive von Lehrkräften und Schülerinnen und Schüler" (Lenski et al., 2015) als Adressaten des Unterrichts und durchaus als Experten hinsichtlich der Wirkung pädagogischer Prozesse sei auf die interessante Diplomarbeit zur „Unterrichtsqualität aus der Schülerperspektive" (Piskol, 2008) hingewiesen.

4 Effektstärke: Cohens $d$ stellt die Effektstärke für Mittelwertunterschiede dar und wird zur Beurteilung der praktischen Relevanz signifikanter Ergebnisse herangezogen. Bananimation erklärt auf https://youtu.be/oqNHzMXN0u4 Was ist eine Effektstärke?

5 Informationen zum „Projekt Kategoriensystem zur überfachlichen Aufgabenanalyse" finden sich auf der Homepage: http://www.erziehungswissenschaft.unituebingen.de/abteilungen/schulpaedagogik/aufgabenanalyse.html 


\section{Lernstand - Lernwirksamkeit - Lernleistung}

\section{Literatur}

Artelt, C. \& Rausch, T. (2014). Accuracy of teacher judgements. When and for what reasons? In S. Krolak-Schwerdt, S. Glock \& M. Böhmer (Hrsg.), Teachers' Professional Development. Assessment, Training, and Learning (S. 25-44). Rotterdam: Sense. http://dx.doi.org/10.1007/978-94-6209-536-6_3

Beck, E., Baer, M., Guldimann, T., Bischoff, S., Brühwiler, P., Müller, P., Niedermann, R., Rogalla, M. \& Voigt, F. (2008). Adaptive Lehrkompetenz. Analyse und Struktur, Veränderung und Wirkung handlungssteuernden Lehrerwissens. Münster: Waxmann.

Berliner, D. C. (2005). The near impossibility of testing for teacher quality. Journal of Teacher Education, 56(3), 205-213. http://dx.doi.org/10.1177/0022487105275904

Blömeke, S., Gustafsson, J.-E. \& Shavelson, R. (2015). Beyond dichotomies: Viewing competence as a continuum. Zeitschrift für Psychologie, 223(1), 3-13. http://dx.doi.org/10.1027/2151-2604/a000194

Bohl, T., Drüke-Noe, C., Hoppe, H., Kleinknecht, M. \& Maier, U. (2015). Was bringt diese Aufgabe? Pädagogik, 67(5), 28-31.

Brandl, W. (2012). Begriffe - Konzepte - Argumente: Bausteine für mentale Konstruktionsprozesse im kompetenzorientierten Unterricht. Haushalt in Bildung \& Forschung, 1(3), 31-51.

Breidenstein, G. (2015). Zensuren: Anwendungsgebiete, Risiken und Nebenwirkungen. In S. G. Huber (Hrsg.), Jahrbuch Schulleitung 2015. Befunde und Impulse zu den Handlungsfeldern des Schulmanagements (S. 60-68). Neuwied: Luchterhand/WoltersKluwer.

Brosziewski, A. (2010). Von Bildung zu Kompetenz. Semantische Verschiebungen in den Selbstbeschreibungen des Erziehungssystems In T. Kurtz \& M. Pfadenhauer (Hrsg.), Soziologie der Kompetenz (S. 119-134) Wiesbaden: Springer VS. http://dx.doi.org/10.1007/978-3-531-91951-5_7

Brunner, R. \& Zeltner, W. (1980). Lexikon zur Pädagogischen Psychologie und Schulpädagogik. München: Ernst Reinhardt.

Deterding, S., Khaled, R., Nacke, L. E. \& Dixon, D. (2011). Gamification: Toward a definition. CHI 2011, May 7-12, 2011, Vancouver, BC, Canada.

http://gamification-research.org/wp-content/uploads/2011/04/02-DeterdingKhaled-Nacke-Dixon.pdf

Eckart, E. \& Schratz, M. (2015). Differenzierung - ein amodernes Konzept? Vom „Lehrseits“ zum „Lernseits“. Erwägen Wissen Ethik, 26(2), 193-195.

Felten, M. \& Stern, E. (2012). Lernwirksam unterrichten. Im Schulalltag von der Lernforschung profitieren. Berlin: Cornelsen Scriptor. 


\section{Lernstand - Lernwirksamkeit - Lernleistung |}

Fleckenstein, J., Zimmermann, F, Köller, O. \& Möller, J. (2015). What works in school? Expert and novice teachers' beliefs about school effectiveness. Frontline Learning Research, 3(2), 27-46. http://dx.doi.org/10.14786/flr.v3i2.162

Fleischer, J., Koeppen, K., Kenk, M., Klieme, E. \& Leutner, D (2013). Kompetenzmodellierung: Struktur, Konzepte und Forschungszugänge des DFG- Schwerpunktprogramms. Zeitschrift für Erziehungswissenschaft, 16, 5-22. http://dx.doi.org/10.1007/s11618-013-0379-z

Gabler Wirtschaftslexikon. Stichwort: Gamification. http://wirtschaftslexikon.gabler.de/Archiv/688938796/gamification-v4.html

Geier, I. (2015). Neue Lernformen - Neue Lehrkultur. ph.script 9 (Neue Lehr- und Lernformen), 9-17.

http://www.phsalzburg.at/fileadmin/PH_Dateien/PH-Script/phscript09_web.pdf

Gruschka, A. (2007). „Was ist guter Unterricht?“. Über neue AllgemeinModellierungen aus dem Geiste der empirischen Unterrichtsforschung. Pädagogische Korrespondenz, 36, 10-43. URN: urn:nbn:de:0111-opus-79644

Hattie, J. (2009). Visible learning: A synthesis of over 800 meta-analyses on achievement. Abington: Routledge.

Hattie, J. \& Zierer, K. (2016). Betrachte Lernen als harte Arbeit! Pädagogik, 68(9), 44-48.

Helmke, A. (2015). Unterrichtsqualität und Lehrerprofessionalität: Diagnose, Evaluation und Verbesserung des Unterrichts (6., akt. Aufl.). Seelze: KlettKallmeyer.

Helmke, A. \& Lenske, G. (2015). Lernwirksamkeit des Lehrerhandelns - Forschungsergebnisse und Diagnostik. In S. G. Huber (Hrsg.), Jahrbuch Schulleitung 2015. Befunde und Impulse zu den Handlungsfeldern des Schulmanagements (S. 253-268). Neuwied: Luchterhand/WoltersKluwer.

Helmke, A. \& Schrader, F. (2016). Angebots-Nutzungs-Modell der Wirkfaktoren akademischer Leistungen. In M. A. Wirtz (Hrsg.), Dorsch-Lexikon der Psychologie.

https://portal.hogrefe.com/dorsch/angebots-nutzungs-modell-der-wirkfaktorenakademischer-leistungen

Helsper, W. \& Klieme, E. (2013). Quantitative und qualitative Unterrichtsforschung - eine Sondierung. Einführung in den Thementeil. Zeitschrift für Pädagogik, 59(3), 283-290. URN: urn:nbn:de:0111-pedocs-119381

Heymann, H. W. (2015). Warum sollte Unterricht „kognitiv aktivieren“? Anregung von vertiefendem, verstehendem, vernetzendem Lernen. Pädagogik, 67(5), 6-9.

Holzberger, D. \& Kunter, M. (2016). Unterricht aus der Perspektive der Pädagogischen Psychologie und der empirischen Unterrichtsforschung. In J. Möller, M, Köller \& T. Riecke-Baulecke (Hrsg.), Basiswissen Lehrerbildung: Schule und Unterricht - Lehren und Lernen (S. 39-52). Seelze: Klett Kallmeyer. 


\section{Lernstand - Lernwirksamkeit - Lernleistung}

Huber, S. G. (Hrsg.) (2015). Jahrbuch Schulleitung 2015. Befunde und Impulse zu den Handlungsfeldern des Schulmanagements. Schwerpunkt: lernwirksam unterrichten und fördern. Neuwied: Luchterhand/WoltersKluwer.

Institut für Qualitätsentwicklung (IQ). (Hrsg.) (2010). Fragebögen zur Unterrichtsqualität (iq Praxis 8; 2., überarb. Fassung). Wiesbaden: Institut für Qualitätsentwicklung.

Institut für Qualitätsentwicklung (IQ). (Hrsg.) (2011). Hessischer Referenzrahmen Schulqualität. Qualitätsbereiche, Qualitätsdimensionen und Qualitätskriterien. Wiesbaden: Institut für Qualitätsentwicklung.

Kipmann, U. (2015). Neue Lehr- und Lernformen - Was wissen PH-Studierende zu diesem Thema. ph.script 9 (Neue Lehr- und Lernformen), 82-87.

http://www.phsalzburg.at/fileadmin/PH_Dateien/PH-Script/phscript09_web.pdf

Kleinknecht, M., Maier, U., Metz, K., \& Bohl, T. (2011). Analyse des kognitiven Aufgabenpotenzials. Unterrichtswissenschaft, 39(4), 328-343.

Köller, O. (2012). What works best in school? Hatties Befunde zu Effekten von Schul- und Unterrichtsvariablen auf Schulleistungen. Psychologie in Erziehung und Unterricht, 59(1), 72-78. http://dx.doi.org/10.2378/peu2012.art06d

Kunter, M. \& Ewald, S. (2016). Bedingungen und Effekte von Unterricht: Aktuelle Forschungsperspektiven aus der pädagogischen Psychologie. In N. McElvany, W. Bos, H. G. Holtappels, M. M. Gebauer \& F. Schwabe (Hrsg.), Bedingungen und Effekte guten Unterrichts (Dortmunder Symposium der Empirischen Bildungsforschung, S. 9-32). Münster: Waxmann.

Kunter, M. \& Klusmann, U. (2010). Kompetenzmessung bei Lehrkräften - Methodische Herausforderungen. Unterrichtswissenschaft, 38(1), 86-86.

Kunter, M. \& Trautwein, U. (2013). Psychologie des Unterrichts. Paderborn: Schöningh.

Kunter, M., Seidel, T. \& Artelt, C. (2015). Editorial „Pädagogisch-psychologische Kompetenzen von Lehrkräften“. Zeitschrift für Entwicklungspsychologie und Pädagogische Psychologie, 47(2), 59-61. http://dx.doi.org/10.1026/0049-8637/a000123

Lenski, A. E., Richter, D. \& Pant, H. A. (2015). Kompetenzorientierung im Unterricht aus der Perspektive von Lehrkräften und Schülerinnen und Schüler. Zeitschrift für Pädagogik, 61(5), 712-737.

Leuders, T. \& Sodian, B. (2013). Inwiefern sind Kompetenzmodelle dazu geeignet kognitive Prozesse von Lernenden zu beschreiben? Zeitschrift für Erziehungswissenschaft, 16, 27-33.

Lipowsky, F. (2006). Auf den Lehrer kommt es an. Empirische Evidenzen für Zusammenhänge zwischen Lehrerkompetenzen, Lehrerhandeln und dem Lernen der Schüler. Zeitschrift für Pädagogik (51. Beiheft), 47-70.

URN: urn:nbn:de:0111-opus-73704 


\section{Lernstand - Lernwirksamkeit - Lernleistung |}

Lipowsky,, F. (2015). Unterricht. In E. Wild \& J. Möller (Hrsg.), Pädagogische Psychologie (S. 69-105). Heidelberg: Springer.

http://dx.doi.org/10.1007/978-3-642-41291-2_4

Maier, U., Kleinknecht, M., Metz, K. \& Bohl, T. (2010). Ein allgemeindidaktisches Kategoriensystem zur Analyse des kognitiven Potenzials von Aufgaben. Beiträge zur Lehrerinnen- und Lehrerbildung, 28(1), 84-96.

https://www.bzl-online.ch/archivdownload/artikel/BZL_2010_1_84-96.pdf

Minnameier, G., Hermkes, R. \& Mach, H. (2015). Kognitive Aktivierung und Konstruktive Unterstützung als Prozessqualitäten des Lehrens und Lernens. Zeitschrift für Pädagogik, 61(6), 837-856.

Nohl, A.-M., von Rosenberg, F. \& Thomsen, S. (2015). Bildung und Lernen im biographischen Kontext. Empirische Typisierungen und praxeologische Reflexionen. Wiesbaden: Springer VS.

Piskol, K. (2008). Unterrichtsqualität aus der Schülerperspektive: Ein Verfahren zur Unterrichtsentwicklung (Diplomarbeit). Universität Mannheim. https://ub-madoc.bib.uni-mannheim.de/2664/1/Piskol_2008.pdf

Prediger, S. \& Scherres, C. (2012). Niveauangemessenheit von Arbeitsprozessen in selbstdifferenzierenden Lernumgebungen. Journal für Mathematik-Didaktik, 33(1), 143-173. http://dx.doi.org/10.1007/s13138-012-0035-9

Rausch, T., Matthäi, J. \& Artelt, C. (2015). Mit Wissen zu akkurateren Urteilen? Zum Zusammenhang von Wissensgrundlagen und Urteilsgüte im Bereich des Textverstehens. Zeitschrift für Entwicklungspsychologie und Pädagogische Psychologie 47(3),147-158. http://dx.doi.org/10.1026/0049-8637/a000124

Renkl, A. (2016). Multiple Ziele in Unterricht und Lernumgebungen: Einführung in den Thementeil. Unterrichtswissenschaft, 44(3), 206-210.

Retelsdorf, J. \& Möller, J. (2016). Diagnostik und Leistungsbeurteilung. In J. Möller, M, Köller \& T. Riecke-Baulecke (Hrsg.), Basiswissen Lehrerbildung: Schule und Unterricht - Lehren und Lernen (S. 23-38). Seelze: Klett Kallmeyer.

Schratz, M. (2009). „Lernseits“ von Unterricht. Alte Muster, neue Lebenswelten was für Schulen? Lernende Schule, 12(46/47), 16-21.

Seidel, T. (2014). Angebots-Nutzungs-Modelle in der Unterrichtspsychologie. Integration von Struktur- und Prozessparadigma. Zeitschrift für Pädagogik, 60(6), 850-866.

Südkamp, A., Kaiser, J. \& Möller, J. (2012). Accuracy of teacher' judgements of students' academic achievement: A meta-analysis. Journal of Educational Psychology, 104(3), 743-762. http://dx.doi.org/10.1037/a0027627

Südkamp, A., Kaiser, J. \& Möller, J. (2014). Teachers' judgments of students' academic achievement: Results from field and experimental studies. In S. Krolak-Schwerdt, S. Glock \& M. Böhmer (Hrsg.), Teachers' Professional Development. Assessment, Training, and Learning (S. 5-25). Rotterdam: Sense. http://dx.doi.org/10.1007/978-94-6209-536-6_2 


\section{Lernstand - Lernwirksamkeit - Lernleistung}

Terhart, E. (2011). Has John Hattie really found the holy grail of research on teaching? An extended review of Visible Learning. Curriculum Studies, 43(3), 425-438. http://dx.doi.org/10.1080/00220272.2011.576774

Wecker, C., Hetmanek, A. \& Fischer, F. (2016). Zwei Fliegen mit einer Klappe? Fachwissen und fächerübergreifende Kompetenzen gemeinsam fördern. Unterrichtswissenschaft, 44(3), 226-238.

Weinert, F. E. (Hrsg.) (2014). Leistungsmessung in Schulen (3. Aufl.). Weinheim: Beltz.

Zierer, K. (2016). Hattie für gestresste Lehrer. Kernbotschaften und Handlungsempfehlungen aus John Hatties Visible Learning und Visible Learning for Teachers (2. Aufl.). Baltmannsweiler: Schneider Verlag.

\section{Verfasser}

Werner Brandl M.A.

Institutsrektor

Staatsinstitut für die Ausbildung von Fachlehrern

- Abteilung II -

Am Stadtpark 20

D-81243 München

E-Mail: wbrandl@stif2.de

Internet: www.stif2.de 